\title{
Visuomotor Behaviors in Larval Zebrafish after GFP-Guided Laser Ablation of the Optic Tectum
}

\author{
Tobias Roeser and Herwig Baier \\ Department of Physiology and Programs in Neuroscience, Genetics, Developmental Biology, and Human Genetics, University of California at San Francisco, \\ San Francisco, California 94143
}

\begin{abstract}
The optic tectum is the largest visual center in most vertebrates and the main target for retinal ganglion cells (RGCs) conveying visual information from the eye to the brain. The retinotectal projection has served as an important model in many areas of developmental neuroscience. However, knowledge of the function of the tectum is limited. We began to address this issue using laser ablations and subsequent behavioral testing in zebrafish. We used a transgenic zebrafish line that expresses green-fluorescent protein in RGCs projecting to the tectum. By aiming a laser beam at the labeled retinal fibers demarcating the tectal neuropil, the larval tectum could be selectively destroyed. We tested whether tectum-ablated zebrafish larvae, when presented with large-field movements in their surroundings, displayed optokinetic responses (OKR) or optomotor responses (OMR), two distinct visuomotor behaviors that compensate for self-motion. Neither OKR nor OMR were found to be dependent on intact retinotectal connections. Also, visual acuity remained unaffected. Tectum ablation, however, slowed down the OKR by reducing the frequency of saccades but left tracking velocity, gain, and saccade amplitude unaffected. Removal of the tectum had no effect on the processing of second-order motion, to which zebrafish show both OKR and OMR, suggesting that the tectum is not an integral part of the circuit that extracts higher-order cues in the motion pathway.
\end{abstract}

Key words: visual system; vision; retina; optomotor; optokinetic; behavior; retinal ganglion cell; tectum; superior colliculus; zebrafish; Danio rerio; transgenic; GFP; sonic hedgehog; laser ablation

\section{Introduction}

Over the past decades, the retinotectal projection has served as a prominent model, in which mechanisms of axon guidance and topographic map formation were discovered (Holt and Harris, 1998). More recently, after demonstration of activity-dependent rearrangements of the retinotopic map (Debski and Cline, 2002) and of rapid plasticity at the retinotectal synapse (Zhang et al., 1998), the role of visual experience in shaping tectal response properties has become a subject of intense investigation (Engert et al., 2002; Sin et al., 2002). This emphasis on the retinotectal pathway owes to the fact that the tectum is large and well accessible and receives the vast majority of retinal ganglion cell axons conveying visual information from the eye to the brain. Comparatively little is known, however, about the function of the tectum. Various lesioning studies have been performed in adult animals and have established that the tectum is involved in complex behavioral tasks, such as orientation toward prey and away from predators (see Discussion). However, these studies have not been

\footnotetext{
Received Dec. 26, 2002; revised Feb. 19, 2003; accepted Feb. 24, 2003.

This study was funded by a Sandler investigator award, a David and Lucile Packard Fellowship, an Alfred P. Sloan Fellowship, and National Institutes of Health Grant R01-EY12406 (H.B.). T.R. was supported by a DoktorandenStipendium from the Boehringer Ingelheim Fonds. We thank the following people: Ethan Gahtan for contributing confocal images (Fig. 3); Laszlo Bocskai in the physiology machine shop for assistance in building the OKR setup; Carl Neumann for the Shh:GFP transgenic line; Akira Muto for contributing the moments algorithm to the eye movement analysis program; Karl Götz, Ann B. Butler, Mario Wullimann, and Steve Lisberger for advice; all members of our laboratory, in particular Ethan Gahtan and Matt Smear, for helpful discussions or comments on this manuscript; and Ira Herskowitz, Steve Hauser, and the Sandler family for generous support. T.R. thanks Cornelia Fricke for support.

Correspondence should be addressed to Herwig Baier, University of California at San Francisco, Department of Physiology, 513 Parnassus Avenue, S-762, San Francisco, CA 94143-0444. E-mail: hbaier@itsa.ucsf.edu.

T. Roeser's present address: Isenbruck and Partners, Patent Attorneys, Technologiepark Heidelberg Biopark, Im Neuenheimer Feld 582, 69120 Heidelberg, Germany.

Copyright $\odot 2003$ Society for Neuroscience $\quad$ 0270-6474/03/233726-09\$15.00/0
}

able to elucidate which features in a visual scene tectal neurons process and how the tectum is integrated in the circuits producing even simple visually mediated behaviors. This information is critical if the development of the retinotectal system is ever to be linked to the neural circuits underlying behavior of the animal.

Vertebrates exhibit numerous reflex behaviors that are driven by visual stimuli. In zebrafish and other teleosts, two prominent visuomotor responses have been described, the optokinetic response (OKR) and the optomotor response (OMR). Both probably serve to compensate for self-motion. The OKR (see Fig. 1A) encompasses smooth eye movements, which track moving patterns, and rapid reset movements, or saccades. The OMR (see Fig. $1 \mathrm{~B}$ ) is a reflexive swimming in the direction of perceived motion. Fish use the OMR to maintain a stable position in a flowing river by responding to the apparent movement of visual cues present in the riverbed. Because both OKR and OMR can be reliably evoked in the laboratory at early larval stages, these behaviors have been used in genetic screens to identify mutations disrupting development and function of the visual system (Brockerhoff et al., 1995; Neuhauss et al., 1999; Gnügge et al., 2001; Kay et al., 2001).

Despite the recent surge of interest in the OKR and OMR, it is not known where in the zebrafish brain, downstream of the retina, large-field motion is processed. It is likely that dedicated pathways exist for these hard-wired responses, but the neural substrates have not been identified in fish. In zebrafish larvae, as early as $4 \mathrm{~d}$ post-fertilization ( $4 \mathrm{dpf}$ ), retinal ganglion cells (RGCs) project their axons to 10 different visual areas, referred to as "arborization fields" (AFs) (Burrill and Easter, 1994). Shortly after assembly of the retinofugal pathways, robust OKR and OMR can be evoked (Easter and Nicola, 1996; Neuhauss et al., 
1999). A classical study in adult goldfish surgically removed the tectum and found that this operation abolished the OMR but not the OKR (Springer et al., 1977). We now revisited this question in larval zebrafish using an improved lesioning technique, green fluorescent protein (GFP)-guided laser ablation.

Psychophysicists often distinguish between first-order (Fourier) and second-order (non-Fourier) motion stimuli. Firstorder motion is defined by dynamic modulations of luminance that are visible to Reichardt's classical motion detectors (said to be sensitive to Fourier motion energy; see Adelson and Bergen, 1985). Second-order motion, on the other hand, is not defined by luminance modulations but rather by modulations of higherorder features, such as local contrast, flicker, or local motion (Chubb and Sperling, 1988; Cavanagh and Mather, 1989). Psychophysical and lesion studies in humans have suggested that second-order motion processing occurs in the cortex (Baker, 1999) and that first- and second-order stimuli may be processed by different cortical pathways (Wilson et al., 1992; Ledgeway and Smith, 1994; Lu and Sperling, 1995; Vaina and Cowey, 1996). Zebrafish detect second-order motion without a cortex (Orger et al., 2000). Our ablation experiments allowed us to test whether an intact tectum is necessary for the computation of higher-order motion cues.

\section{Materials and Methods}

Transgenic Shh:GFP zebrafish. Zebrafish of the TL strain were kept and bred according to standard procedures. Less than 40 larvae were kept in one $9 \mathrm{~cm}$ Petri dish, and the medium was cleaned daily. To visualize the tectum for laser ablation, we used the Shh:GFP transgenic line (Neumann and Nüsslein-Volhard, 2000). The GFP reporter is expressed early in most, or all, RGCs and is present in retinal axons traveling to the tectum and other retinorecipient areas. Thus, GFP demarcates the location and extent of the tectal neuropil in vivo and provides information about the success of laser ablation (see below). The GFP signal is best imaged with a confocal laser-scanning microscope (MRC-1024; Bio-Rad, Hercules, CA), but the outline of the tectum is well visible under either a compound microscope (Axioskop II; Zeiss, Oberkochen, Germany) or an epifluorescence-equipped dissecting microscope (MZ FLIII; Leica, Nussloch, Germany). In pilot experiments, we determined that transgenic fish are indistinguishable from nontransgenic fish with respect to their visually mediated behavior.

Laser ablations. Hemizygous GFP-expressing fish larvae were sorted on day 3. On day 6 or 7, they were transferred to a depression slide and embedded, dorsal side up, in $1.5 \%$ methyl cellulose in embryo rearing solution. The medium was supplemented with MESAB (3-aminobenzoic acid ethyl esther methane sulfonate) (Sigma, St. Louis, MO) to anesthetize the fish. Larvae were kept in the medium for no more than $20 \mathrm{~min}$ to avoid harm by prolonged anesthesia. Laser ablations were performed using a MicroPoint (Photonic Instruments, Arlington Heights, IL) laser system (equipped with a VSL-337ND-S nitrogen laser; Laser Science, Franklin, MA) on a Zeiss Axioskop II compound microscope. The primary UV laser pumped a $440 \mathrm{~nm}$ Coumarin laser, which was aimed via a $20 \times$ microscope lens (Zeiss Plan-Neofluar $20 \times$, numerical aperture 0.5 ) onto the labeled fibers in the tectum. The laser irradiation was performed under simultaneous visual control through GFP optics. Laser power was attenuated with neutral gray filters to avoid excessive tissue damage. In our hands, the laser power was optimal when single laser pulses caused slight movement of the targeted tissue and, occasionally, small air bubbles. The laser was used at a pulse rate of $2-10 \mathrm{~Hz}$. During the ablation procedure, the fish were repositioned to aim the laser beam onto the (arched) tectum from an almost perpendicular angle. Under these conditions, ablation of one tectal lobe took between 5 and $12 \mathrm{~min}$.

For OKR experiments (Fig. 1A), one tectal lobe was ablated. The contralateral tectum served as an intrinsic control. Larvae were allowed to recover for 3-6 hr before their OKR was tested. This interval did not leave enough time for regeneration of retinotectal fibers but was sufficiently

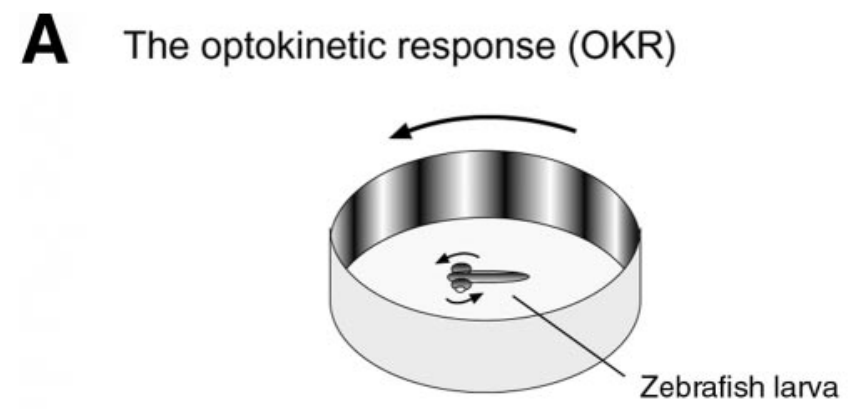

\section{B The optomotor response (OMR)}

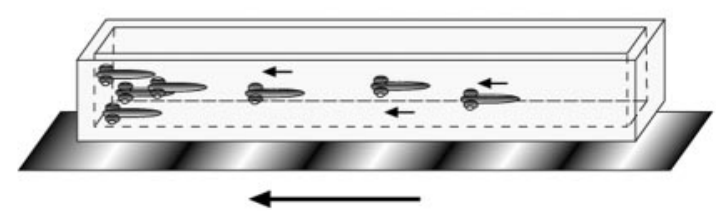

Figure 1. Schematic depiction of the two visuomotor behaviors investigated in this study. $A$, During the OKR, immobilized fish respond with eye movements to a moving stimulus in their surroundings. $B$, During the OMR, fish swim to follow a moving stimulus displayed on the bottom of their tank. The direction of motion is indicated by arrows.

long for recovery from anesthesia (i.e., the fish swam around normally and responded to touch or tone stimuli). For OMR experiments (Fig. $1 B$ ), bilateral ablations were performed on 2 consecutive days. This protocol allowed for intermittent recovery from anesthesia and laser treatment, thus improving the survival rates of the operated animals. The OMR was scored 3-6 hr after the second laser session. For each experiment, transgenic clutch mates of the experimental group were subjected to a "sham operation." These control fish were mounted in methyl cellulose, imaged under the fluorescence microscope, along with their tectum-ablated siblings, and subjected to laser ablation of a few head melanophores.

OKR stimulation. A liquid crystal display projector (In Focus LP 550) pointed upward into a white paper drum $(50 \mathrm{~mm}$ height, $56 \mathrm{~mm}$ inner diameter) that rested on a transparent glass plate (Fig. $2 \mathrm{~A}$ ). A wide-angle conversion lens (Kenko VC-050Hi) and a close-up lens (King CU + 1) were inserted between the projector and the drum to focus the projector light into the drum. A neutral gray filter (Contax ND4) and an iris (12 $\mathrm{mm}$ diameter) were used to reduce the amount of light and increase the depth of focus. Zebrafish larvae were placed in an inverted lid of a $40 \mathrm{~mm}$ Petri dish on top of the glass plate and immobilized in $2 \%$ methyl cellulose. The dish was shielded from the direct beam of the projector by a diffusor.

Stimuli were generated on a laptop computer using plug-ins written for the public domain software NIH Image/J. The original image was a luminance-modulated spoked wheel, which was transformed into a set of vertical stripes by the projection process (Fig. $2 A$ ). Movement of the grating was generated by computer-animated rotation of the wheel. Stimulus velocity for the sine-wave gratings was $20^{\circ}$ /sec. If stimuli were presented to one eye of the fish only, the grating was restricted to $180^{\circ}$ of the visual field, whereas the other half of the visual field was illuminated with a uniform gray stimulus.

Second-order motion. To generate motion-defined motion, a random dot pattern was shown to the fish in which the rows of dots were animated vertically. Speed and direction of the vertical movement were defined by a sine function, the "motion envelope," with a spatial frequency of $90^{\circ}$ (Fig. $2 B$ ). In each subsequent frame, the motion envelope is shifted horizontally by $3^{\circ}$, changing the speed and the direction of the vertical movements accordingly. This shifting creates the illusion of horizontal motion, despite the fact that the individual dots are not moving sideways but only up or down. The velocity of the motion envelope was 
$90^{\circ} / \mathrm{sec}$ (30 frames per second). The size of the dots was $3^{\circ}$. The amplitude of the motion envelope was $48^{\circ}$. This amplitude corresponds to the maximum distance that an individual dot travels vertically, before its direction is reversed.

OKR analysis. Eye movements of individual fish were recorded at four frames per second by an overhead CCD camera (High Precision Color CCD, 8215-1300; Cohu, San Diego, CA), mounted on a Nikon (Tokyo, Japan) SMZ 800 dissecting scope, and stored as digital movies using a Scion (Frederick, MD) frame grabber board. These movies were analyzed using a Java program written as a plug-in for the NIH Image/J image analysis software. The eyes of zebrafish larvae are darkly pigmented, in contrast to their head and trunk, which contain only scattered melanophores and are practically translucent. To keep the image of shape and size of the eyes between individuals constant, fish were mounted consistently dorsal side up. Our program tracked the eyes as the largest dark, ellipsoid particles in a thresholded image (Fig. 2C) and measured their angle with an accuracy of approximately $\pm 2^{\circ}$, relative to the body axis, in each frame (Fig. 2D). Eye movements were plotted as angle over time, and the saccade rate was measured manually in representative parts of the trace (Fig. $2 E$ ). To determine the gain of the OKR, the peak velocity of eye movements was measured, which is usually during the first two-thirds of the tracking phase. Gain was calculated by dividing tracking velocity by stimulus velocity.

OKR eye movements are conjugate (tracking phases and saccades are synchronized between the eyes), but the amplitudes differ between the eye receiving the stimulus in temporal-nasal direction and the eye that receives the stimulus in nasal-temporal direction (Easter, 1972). Only the eye with the larger amplitudes (presented with the temporal-to-nasal moving stimulus) was analyzed. Statistical significance was determined by a two-tailed $t$ test for paired samples. This test was deemed appropriate, because the two eyes of the same animal were compared (therefore "paired") and because the OKR parameters can change in both directions, i.e., tectum ablation may result in more or less saccades (therefore "two-tailed").

OMR assay. The OMR assay was performed as published previously (Neuhauss et al., 1999; Orger et al., 2000), except that single fish were analyzed. In short, tectum-ablated Shh:GFP transgenic fish and shamtreated controls were individually transferred into $30-\mathrm{cm}$-long and 1-cm-wide Plexiglas tanks on an upward-facing computer monitor. Single fish were placed in the middle of the tank, and a moving sine-wave grating (wavelength of $200^{\circ}$, temporal frequency of $10 \mathrm{~Hz}$ ) was displayed for $30 \mathrm{sec}$. After recording their end positions, the fish (regardless of whether they had moved) were taken up into a plastic transfer pipette and released at their start positions. Each fish underwent eight independent trials, of which the average was taken.

Labeling of the retinofugal projection with DiI. To visualize the entire retinofugal projection, the carbocyanine dye DiI (Molecular Probes, Eugene, OR) was pressure injected (WPI PV-820; World Precision Instruments, Sarasota, FL) into the eye of larvae that had been fixed overnight in $4 \%$ paraformaldehyde in PBS. Fish were embedded in $1 \%$ agarose in half-strength PBS. The dye was dissolved in chloroform to a final concentration of $10 \mathrm{mg} / \mathrm{ml}$. After an overnight diffusion period, DiI labeling
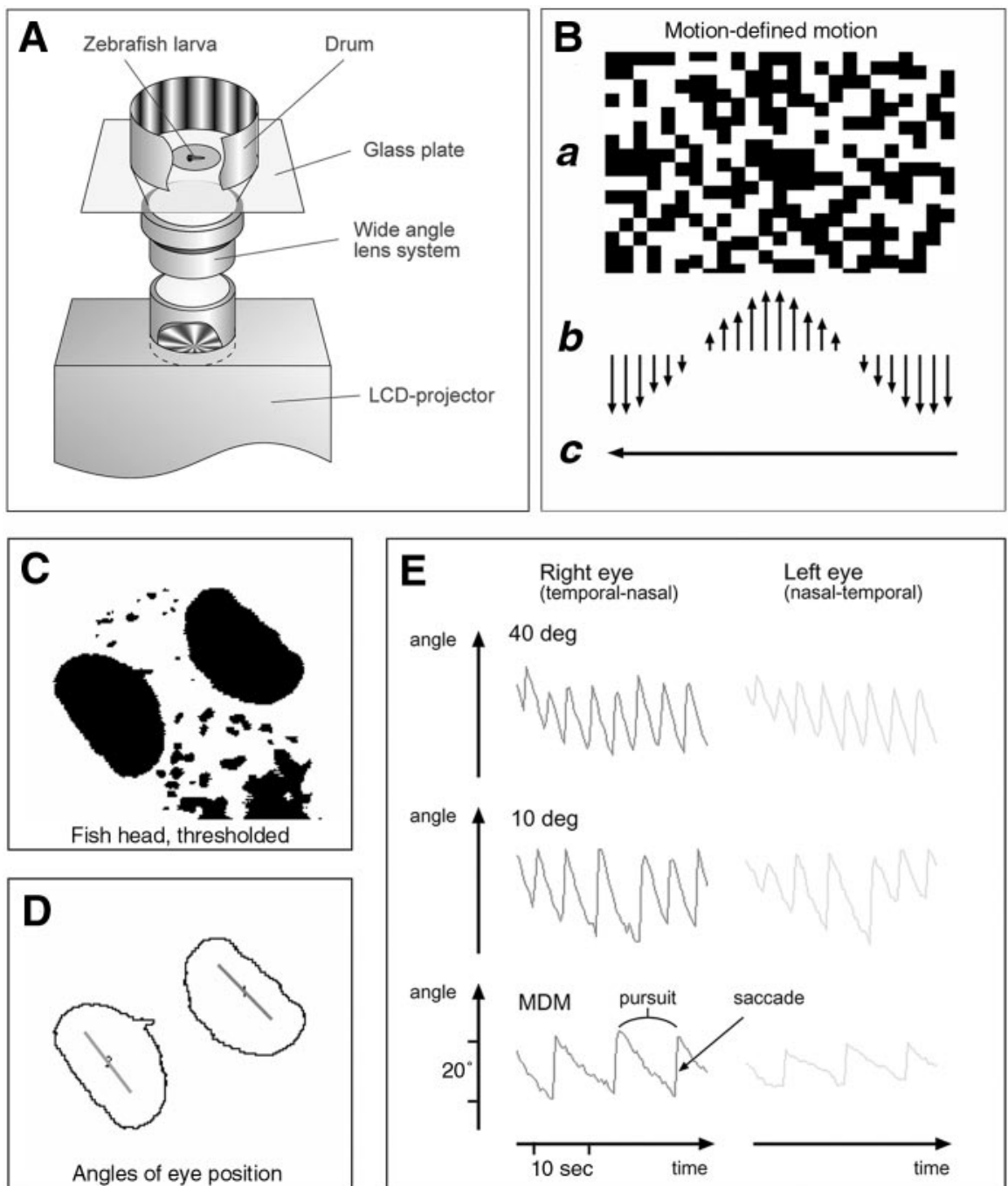

Figure 2. Stimulation and analysis of the OKR. A, OKR stimulation setup. A computer-animated spoked wheel is projected by an LCD-projector onto the inner surface of a white drum, where the spokes are transformed into a vertical grating. Frame-by-frame rotation of the wheel leads to apparent motion of the grating. $B$, Motion-defined motion stimulus. A random dot pattern $(a)$ is envelope $(b)$. In each frame of the movie, the motion envelope is moved horizontally by one row in the direction indicated $(c)$. The in sensation of movement in horizontal direction ( $C$ ), although the dots themselves move only vertically. C, D, Analysis of the 列. wild-type larva during motion of a $40^{\circ}$ sine-wave grating (top), a $10^{\circ}$ sine-wave grating (middle), and motion-defined motion (MDM, see $B$ ). Stimulation in temporal-nasal direction (in this case for the right eye, dark gray) elicits larger amplitudes than stimulation in nasal-temporal direction (left eye, light gray).

was visualized with Texas Red filters (excitation, $540-580 \mathrm{~nm}$; emission, $610 \mathrm{~nm}$ long pass) using the $20 \times$ objective of a Zeiss Axioskop II.

\section{Results}

Targeted laser ablation selectively destroys the retinotectal neuropil

The Shh:GFP reporter transgene (Neumann and NüssleinVolhard, 2000) allows visualization of the tectum in the living zebrafish larva (Fig. 3). Optical sections with a laser-scanning confocal microscope most clearly show the outline of the tectal neuropil (Fig. 3A) and of deeper retinorecipient areas (Fig. 3B), but the structures are also easy to delineate by focusing up and down at low power under the compound microscope. We investigated whether the retinotectal neuropil of GFP transgenic larvae could be ablated by laser irradiation. Fewer than 10 laser pulses 

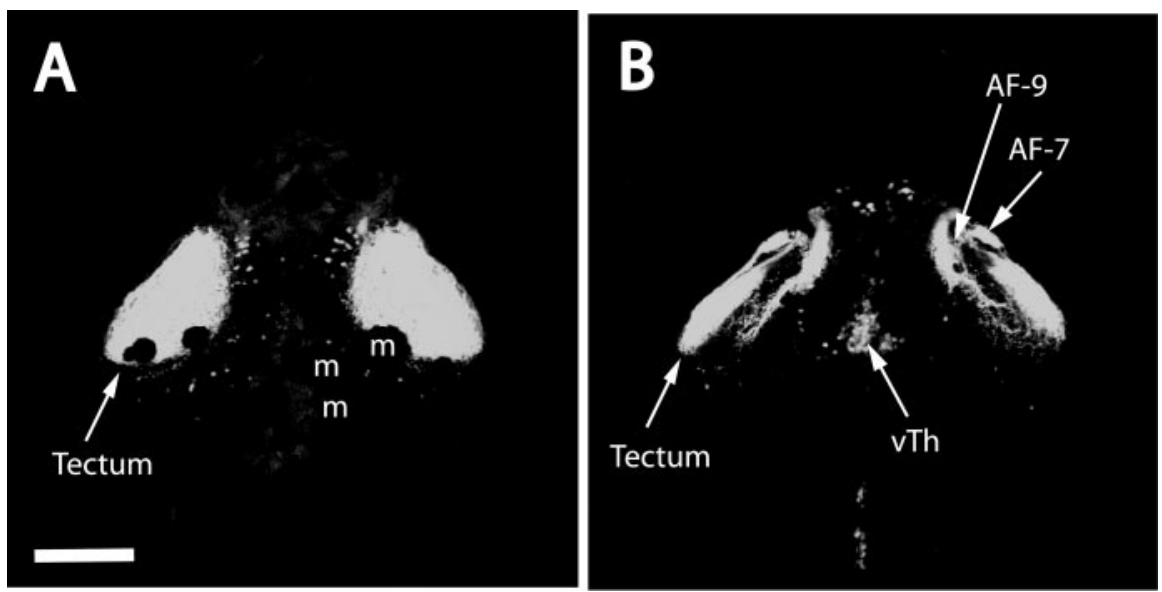

Figure 3. Visualization of the optic tectum in Shh:GFP transgenic fish. A, Dorsal view of the GFP-labeled tectal neuropil in a living, hemizygous, $6 \mathrm{dpf}$ zebrafish larva (projection of several confocal sections). The tectal neuropil is clearly demarcated. A few of the melanophores $(m)$ are indicated. $B$, Deeper optical sections from the same confocal stack. Two additional retinorecipient nuclei (AF-7 and AF-9) are visible now, as well as ventral thalamic cells (vTh), which are also labeled by GFP in this line. The optic chiasm and other retinorecipient arborization fields are positioned more ventrally (data not shown). Scale bar (in $A$ ): $A, B, 100 \mu \mathrm{m}$. not require the tectum (von Frisch, 1911; Dijkgraaf, 1949; Gentle, 1971). Neither unilateral $(n=16)$ nor bilateral $(n=11)$ ablations of the tectal neuropil permanently abolished the VBA (for example, see the fish shown in Fig. 4). Frequently, fish showed expanded melanophores during the first hour after the ablation procedure but recovered before the behavioral tests were conducted. We attribute this temporary darkening to a disturbed stress response to the operation, because hormones, such as epinephrine, are known to also influence body pigmentation in zebrafish. Swimming behavior, postural control, and acoustic startle responses were all qualitatively normal. The combined results confirm that (1) the operation selectively disrupts the tectum and (2) tectum-ablated fish are not blind or otherwise grossly impaired. produced visible damage to the targeted tissue, causing local disruption of the GFP-labeled fibers in a zone of a few micrometers surrounding the laser spot. Ablation of the entire tectal neuropil was accomplished within $\sim 5-12$ min by scanning the tectum with laser pulses. Melanophores (black pigment cells) in the head absorb the laser energy very effectively and burst when hit in passing. Melanophore-ablated transgenic fish, treated alongside the tectum-ablated group on the microscope stage, were used as sham-operated controls in the behavioral experiments.

To ensure that the neuropil had been completely ablated, all fish were inspected after the behavioral testing for the presence or absence of GFP-labeled fibers. Occasionally, we did see GFPfilled axons, mostly in the deep ventrorostral part of the tectum, which had apparently survived the ablation. Data from fish with incomplete ablations were excluded from this study. To further test the success of tectum ablation, operated fish were fixed in $4 \%$ paraformaldehyde in PBS, and the entire retinofugal projection was visualized by filling both eyes with DiI (Fig. 4). On the ablated side, DiI-labeled fibers could be seen arborizing in the pretectum (e.g., arborization fields AF-7 and AF-9) but not in the tectum (Fig. $4 B, D$ ). This indicates that (1) the ablation selectively destroyed the retinotectal neuropil but left the innervation of the pretectal arborization fields and deeper nuclei intact and (2) retinal connections had not regenerated to the tectum during the recovery period. Although the lesion is probably restricted to the tectum and has disconnected it completely from the eye, we are unable to estimate the spread of the damage to unlabeled, deeper parts of the tectum. It is possible that dendrites and somata of tectal cells were also impaired.

As a simple test to control for the selectivity of the laser ablation, we examined whether the visual background adaptation response (VBA) was affected by ablation of the tectum. The VBA is a neuroendocrine camouflage response that matches pigment distribution in the body to ambient light levels (von Frisch, 1911; Hogben and Slome, 1931). Dark-adapted fish disperse their melanin pigment, thus appearing dark, whereas light-adapted fish aggregate the melanin granules, thus appearing pale. Blind fish appear dark all the time, whereas visually impaired fish exhibit gradations of melanin dispersal that are correlated with the degree of their impairment (Neuhauss et al., 1999). Although dependent on the retina as the light-sensing organ, the VBA does

\section{First-order and second-order motion evokes OKR in} larval zebrafish

To measure the OKR of normal and laser-ablated fish, a novel setup was devised that allowed presentation of diverse computergenerated stimuli, including gratings of varying spatial frequency and with second-order characteristics. To obtain baseline data on the OKR in the new setup, we presented moving sine-wave gratings at different wavelengths (5-40 of visual angle per cycle) to unoperated fish (Fig. 5). All gratings were presented at an angular velocity of $20 \% \mathrm{sec}$. The temporal frequency covaries with the spatial frequency of the grating according to the relationship velocity $=($ temporal frequency $) /($ spatial frequency $)$. Saccade rate (Fig. 5A) and tracking velocity (Fig. 5B) of the OKR strongly depended on the stimulus parameters. Tracking velocity was defined as the maximum speed of eye movement, usually during the first two-thirds of the smooth phase. (Toward the end of the smooth phase, the eyes often decelerated significantly.) The strongest responses were observed for 20 and $40^{\circ}$ sine-wave gratings, reaching $\sim 30$ saccades per minute and a tracking velocity of $13.6 \% \mathrm{sec}$. The latter value corresponds to a gain of 0.68 . For finer gratings, both saccade frequency and tracking velocity dropped significantly. For the $7.5^{\circ}$ stimulus, velocity was $5.3^{\circ} / \mathrm{sec}$, corresponding to a gain of 0.22 . No OKR was detectable to a $5^{\circ}$ sinewave grating $(n=5$; data not shown), consistent with the theoretical resolution limit set by the spacing of cone photoreceptors at this age (Easter and Nicola, 1996). In contrast to saccade frequency and tracking velocity, the amplitude of the OKR appeared independent of the stimulus parameters (Fig. 5C). OKR amplitudes were constant for all stimuli, in the range of $20 \pm 2^{\circ}$.

Our previous work showed that OMR is elicited by secondorder motion, i.e., motion devoid of Fourier motion energy and therefore invisible to classical motion detectors (Orger et al., 2000). The new setup enabled us to ask whether second-order motion may also elicit an OKR and if that response depended on an intact tectum. As a test case, we used motion-defined motion, borrowed from the human psychophysical literature (Fig. 2B). To the human observer, such a movie generates the sensation of global horizontal movement in the direction to which the motion envelope is shifted, in addition to local vertical movements (Cavanagh and Mather, 1989). The same stimulus (spatial wavelength of motion envelope, $90^{\circ}$; velocity of second-order motion, 
$90 \%$ sec; dot size, $3^{\circ}$ ) also effectively drove an OKR in zebrafish in the predicted direction $(n>100$; data shown for 12 fish) (Fig. $5 A-C$ ). Saccade rate and gain, but not amplitude, of the OKR were low, despite the low spatial frequency of the stimulus, suggesting that the motion percept was weaker than that produced by a highcontrast first-order stimulus (Fig. $5 A, B$ ). In summary, these experiments demonstrate that (1) the OKR is evoked by both first-order and second-order motion, similar to the OMR and (2) saccade frequency and gain, but not amplitude, of eye movements are reliable quantities that correlate systematically with the spatial frequency of the grating and therefore probably with stimulus saliency.

Saccade frequency of the OKR, but not gain, amplitude, or acuity, are altered by tectum ablation

To test whether the OKR was dependent on the retinotectal projection, unilateral tectum ablations were performed $(n=15$ fish). In this paradigm, stimulation of the eye projecting to the intact tectum serves as an intrinsic negative control because, in zebrafish, the retinotectal projection is completely crossed(Stuermer, 1988; Burrill and Easter, 1994). In all fish tested, vigorous OKR could be elicited by stimulation of either control or ablated side, demonstrating that execution of the OKR per se is not dependent on the tectum (Fig. 6). OKR was also elicited in tectumablated fish by the grating with the highest spatial frequency tested $\left(7.5^{\circ}\right)$. However, we noted significant differences in the frequency of saccades, which were most pronounced at low spatial frequencies $(p<$ 0.01 for the 15 and $40^{\circ}$ stimuli; two-tailed $t$ test for paired samples; $\alpha=0.05$ ) (Fig. 6A). Strikingly, eye tracking velocities during the first phase of the pursuit were not different between control and ablated sides ( $p=0.60$ for the $15^{\circ}$ stimulus; $p=0.93$ for the $40^{\circ}$ stimulus) (Fig. $6 \mathrm{~B}$ ). Furthermore, although there was a slight trend toward larger-amplitude eye movements for the ablated side, this increase $(16 \%)$ was not significant $\left(p=0.12\right.$ for the $15^{\circ}$ stimulus; $p=0.30$ for the $40^{\circ}$ stimulus) (Fig. $6 C$ ). Thus, tectum ablation appeared to slow down the OKR by affecting the pacing of the saccade-generating mechanism and not by affecting gain or amplitude of the OKR.

\section{Second-order motion processing does not require an intact tectum}

Similar to the results for the OKR to first-order stimuli, the response to second-order motion was not qualitatively affected by unilateral tectum ablation (Fig. 6D). All fish responded to stimulation of the ablated side $(n=11)$. The averaged responses of intact and ablated sides were not significantly different. This result demonstrates that second-order motion processing, or at least the processing of motion-defined motion, is not dependent on the optic tectum.
Control
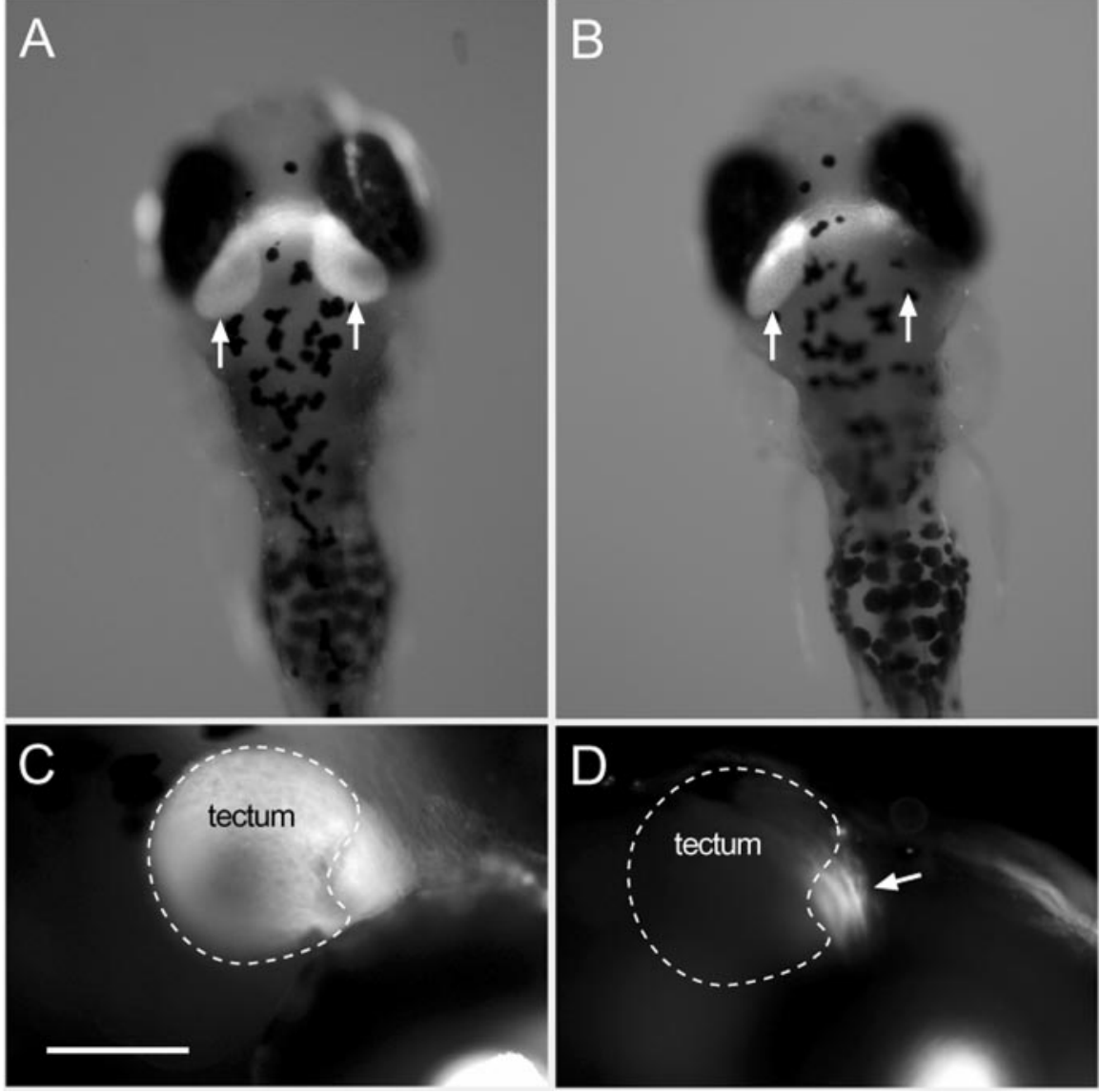

Right tectum ablated

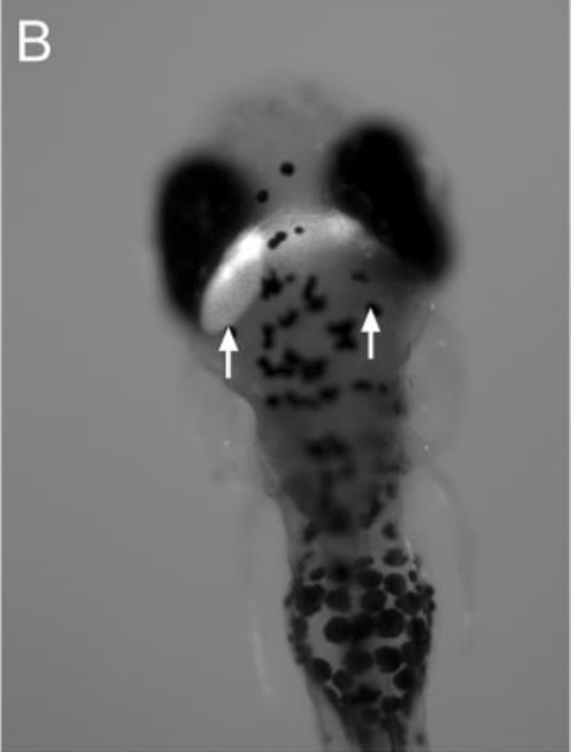

( the laser ablation was assessed, after the behavioral tests, by injection of Dil into both eyes of paraformaldehyde-fixed fish. Dil labels the retinofugal projection, including the tectum. In control fish, both tectal lobes are clearly visible $(A)$, whereas in the tectum-ablated fish, labeled fibers are absent from the ablated tectum $(B) . C, D$, Higher-magnification, dorsolateral views of a control and ablated tectum (dotted line). Anterior is to the right, and posterior to the left. Arrow in D indicates the pretectal arborization field AF-7. Scale bar (in C): C, D, $100 \mu \mathrm{m}$.

\section{The OMR persists after ablation of the tectum and of the pretectal nucleus AF-7}

Bilateral ablations were performed to test whether the OMR was dependent on the retinotectal projection. Most fish showed a vigorous OMR after tectum ablation. The magnitude of the response, as measured by the distances the fish swam in $30 \mathrm{sec}$, was very similar between the two groups (Fig. 7A). These results apparently contradict previous findings in adult goldfish (Springer et al., 1977) (but see Discussion). To test whether the goldfish result could be attributed to lesion of a different nucleus in proximity to the tectum, we performed ablations of AF-7, which is located just rostral to the tectum (Fig. 3B). Most fish showed a reliable and strong OMR after AF-7 ablation and, again, the magnitude of the response appeared unaltered (Fig. $7 B$ ), demonstrating that AF-7 is also not essential for the OMR.

\section{Discussion}

In this study, we laser ablated the retinal input to the optic tectum in zebrafish larvae and investigated the impact of this operation on two visually mediated responses to moving gratings. We discovered that the tectum is required for the correct pacing of saccades during the OKR. The retinorecipient layers of the tectum, however, appear to be dispensable for the execution of both OKR and OMR (contrary to a previous study) and for the processing of second-order motion. 


\section{Completeness and selectivity of the retinotectal ablations}

We confirmed in three ways that the tectal neuropil ablations were selective and encompassed the entire retinal input to the tectum. First, we inspected the GFP labeling pattern after the behavioral tests (to confirm that there were no traces of residual or regenerated innervation in the tectum). Second, we DiIlabeled the entire retinofugal projection after the behavioral tests (to show that the retinotectal connections were selectively disrupted, whereas other projections were intact). And third, we showed that a retina-dependent, but tectum-independent, neuroendocrine response, the visual background adaptation, was unaffected by tectum ablation (confirming that the lesion had not extended beyond the tectum and was not generally impairing vision).

The purpose of our experiments was to remove retinal input to the tectum. Therefore, we targeted the laser only to the GFPlabeled tectal neuropil and not to the deeper layers of the tectum. Because the laser is thought to exert its effect in the tissue by generating heat and free radicals, both of which can spread between cells, it is possible that our method also destroyed the dendrites of tectal cells and, by retrograde degeneration, some of their cell bodies. It is further possible that other connections to and from the tectum were secondarily affected. This is an intrinsic problem of many lesioning techniques, be they surgical, electrolytic, optical, genetic, or by toxin injection. Because the effects of tectum ablations on behavior were quite mild (swimming, control of posture, and acoustic startle were all qualitatively normal), we do not think that the laser treatment disrupted other parts of the CNS around the tectum.

Although we can exclude regeneration of fibers during the time between ablation and behavioral test, it remains formally possible that connections were rearranged after ablation of the tectum. Thus, after the lesion, the function of the tectum in OKR or OMR could be taken over by a different visual area attributable to rewiring of the circuit. However, the intervals between ablation and behavioral test were short: $<24 \mathrm{hr}$ for the OMR experiments and 3-6 hr for the OKR experiments. Also, we should probably expect more dramatic quantitative changes if one of the smaller visual areas were to assume the function of the tectum after lesion. We therefore think that rearrangement, if it occurs at all, is unlikely to lead to almost complete functional recovery within $1 \mathrm{~d}$.

\section{OKR without a tectum}

For our OKR experiments, the neuropil of only one tectal lobe was laser ablated and the corresponding eye was stimulated, whereas the eye ipsilateral to the ablated tectum served as an intrinsic control. This experimental design exploits that the retinotectal projection is completely crossed in zebrafish. We found that execution of the OKR does not require an intact tectum. This result confirms and extends previous lesion studies in monkey, cat, rabbit, frog, and goldfish (Pasik et al., 1966; Lazar, 1973; Collewijn, 1975; Springer et al., 1977) (but see Flandrin and Jeannerod, 1981). In mammals and birds, the nucleus of the optic tract (dorsal terminal nucleus) has been shown to be essential for the horizontal OKR in mammals and birds (Collewijn, 1975; Cazin et al., 1980; Gioanni et al., 1983). A pretectal nucleus with similar electrophysiological properties has been found in frogs (Katte and Hoffmann, 1980; Montgomery et al., 1982; Lazar et al., 1983 ) and possibly in trout (Klar and Hoffmann, 2002). However, no nucleus essential for the OKR has been identified in teleosts so far. Regeneration experiments in goldfish suggest a group of lateral nuclei to be involved in the OKR (Easter et al., 1978). One of the larval retinorecipient fields that may corre-
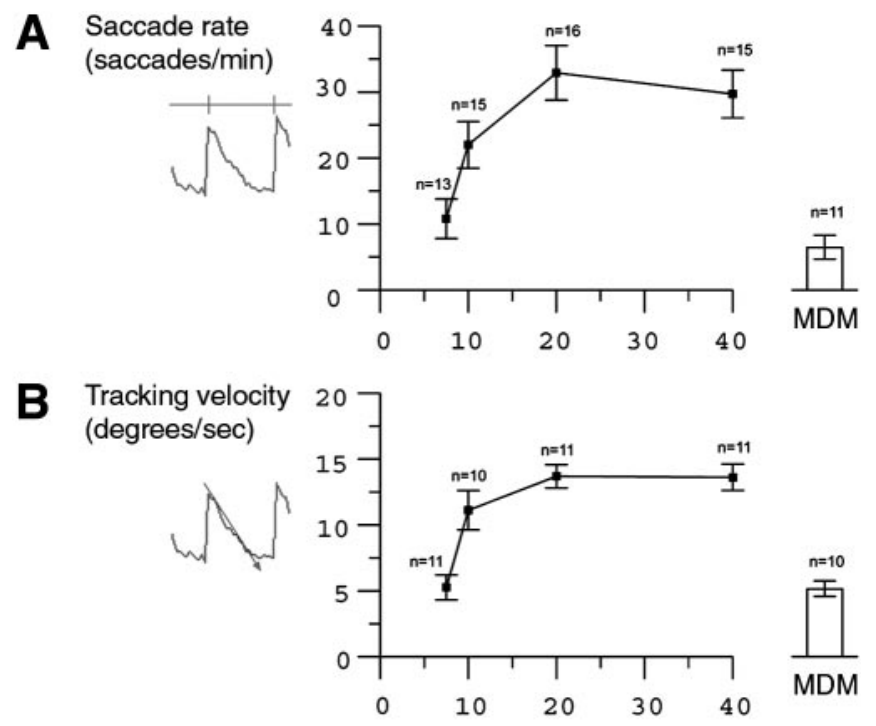

C OKR amplitude

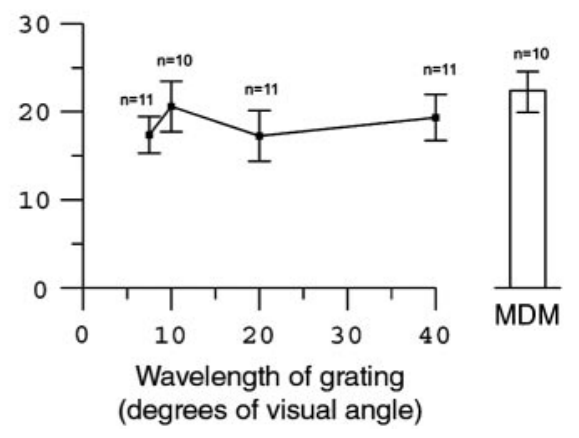

Figure 5. OKR in $7 \mathrm{dpf}$ wild-type zebrafish larvae. Three parameters were tested: saccade rate $(A)$, tracking velocity $(B)$, and OKR amplitude ( $O$. Motion stimuli were sine-wave gratings with wavelengths of $7.5-40^{\circ}$ and constant angular velocity of $20^{\circ} / \mathrm{sec}$, and a motion-defined motion stimulus (MDM; bars on the right). Saccade rate and tracking velocity, but not amplitude, are dependent on the spatial frequency of the stimulus $(A, B)$. The OKR to MDM is clearly detectable but comparatively weak.

spond to these lateral nuclei is AF-6 (Burrill and Easter, 1994). Unfortunately, AF- 6 is very difficult, or impossible, to ablate at 7 dpf without destroying neighboring blood vessels. Preliminary ablations of the adjacent nucleus AF-7 did not suggest a role in the OKR (data not shown).

\section{A role for the tectum in the pacing of the OKR?}

We analyzed three different parameters of the OKR to test for more subtle abnormalities in tectum-ablated zebrafish: the frequency of saccades, the peak tracking velocity (gain), and the amplitude of saccades. Only saccade rate was found to be reduced for tectum-ablated eyes. Gain and saccade amplitude appeared to be unaffected by tectum ablation. Likewise, visual acuity, as measured by OKR to a grating close to the larval fish's resolution limit (Easter and Nicola, 1996), was not diminished. Given the relationship between gain and stimulus saliency (Fig. 5), it appears that tectum ablation does not generally weaken the motion percept. Closer inspection of the OKR traces revealed that the slowing of the OKR reflects mainly a delay in the late part of the pursuit phase, before the saccade resets the eye to its start position. Intriguingly, the mammalian superior colliculus (the homolog of the optic tectum) has also been implicated in the initiation of saccades, at least during normal viewing (Wurtz and Goldberg, 1972; Hikosaka and Wurtz, 1983). The superior colliculus communicates directly with the hindbrain saccade- 
generating circuit (for a recent review, see Scudder et al., 2002). In any case, the larval zebrafish tectum appears to have a facilitating rather than an essential function in the generationof saccades.

\section{OMR without a tectum}

A robust OMR persisted after bilateral tectum ablation. This finding apparently contradicts a classical study that reported that the tectum was required for the OMR in adult goldfish, a related cyprinid species (Springer et al., 1977). Although species and age differences between the two studies cannot be ruled out to account for this discrepancy, we think it is more likely that the difference lies in the extent of the lesions. Our ablation technique left deeper parts of the tectum intact, such as cellbody layers and nonretinal projections. Those parts were removed in the goldfish work. It is conceivable that deeper parts of the tectum play a retina-independent, premotor role in the OMR. Conversely, the lesions performed in goldfish may not have been restricted to the tectum but may have led to secondary degenerations, e.g., attributable to interrupted blood flow. We tested whether a nucleus in close proximity of the tectum, AF-7, was essential for the OMR. Our results indicate that AF-7 is not required for the OMR. However, the ablated tissue in goldfish included the torus longitudinalis (TL) (Springer et al., 1977). The TL lines the dorsomedial edge of the tectum of teleosts (Wullimann, 1994). Intriguingly, the TL is underdeveloped in species that live under conditions in which an OMR is probably unimportant. This group includes bottom-dwellers that are in permanent contact with the substrate, as well as fish species that live on the high seas without stable landmarks (Kishida, 1979). The TL may very well be involved in visual behavior. For instance, the dorsal light response, a tilting of the body axis toward a light source, has been demonstrated to be significantly dependent on the TL in goldfish (Gibbs and Northmore, 1996). However, the TL itself is not retinorecipient, so its participation, if any, in the OMR would be indirect.

\section{Second-order motion processing in the absence of retinotectal connections}

Second-order motion elicits a robust OKR in larval zebrafish. This finding extends previous work from our group, which demonstrated that the OMR in larval zebrafish is evoked by a variety of second-order stimuli (Orger et al., 2000). Humans apparently do not exhibit an OKR in response to second-order motion alone, although second-order features may facilitate a response to first-order motion (Harris and Smith, 1992). Thus, in the human brain, unlike the situation in fish, second-order motion processing appears to be segregated from the control of reflexive eye movements. In addition, the pathways for first-order and second-order motion appear to be distinct in humans. This notion is based on different reaction times and the lack of cross- adaptation between the two systems (Wilson et al., 1992; Ledgeway and Smith, 1994; Lu and Sperling, 1995). Several studies, including one involving a patient with a unilateral lesion (Vaina and Cowey, 1996), have placed the site of second-order motion processing in the visual cortex. If the cortex needs to be invoked to compute non-Fourier movement, then the tectum, as the highest visual center in teleosts, should be the prime candidate for the equivalent function in zebrafish. However, we found that the OKR to second-order motion persists after ablation of the retinotectal connections. Recently, rabbit Y-type RGCs were shown to be able to change their firing rate in response to a second-order motion stimulus (Demb et al., 2001). Thus, retinal circuits may already be in the position to extract both first-order and secondorder motion, and Y cells may feed the information into a single shared channel. Our results do not allow us to delineate the second-order motion pathway in the zebrafish brain, but they exclude an essential role of the retinorecipient layers of the tectum in this process.

\section{Function of the teleost optic tectum}

Electrophysiological and ablation studies from adult teleosts suggest that the tectum is involved in the detection of form and mediates precise orienting responses of body and eyes, the so- 


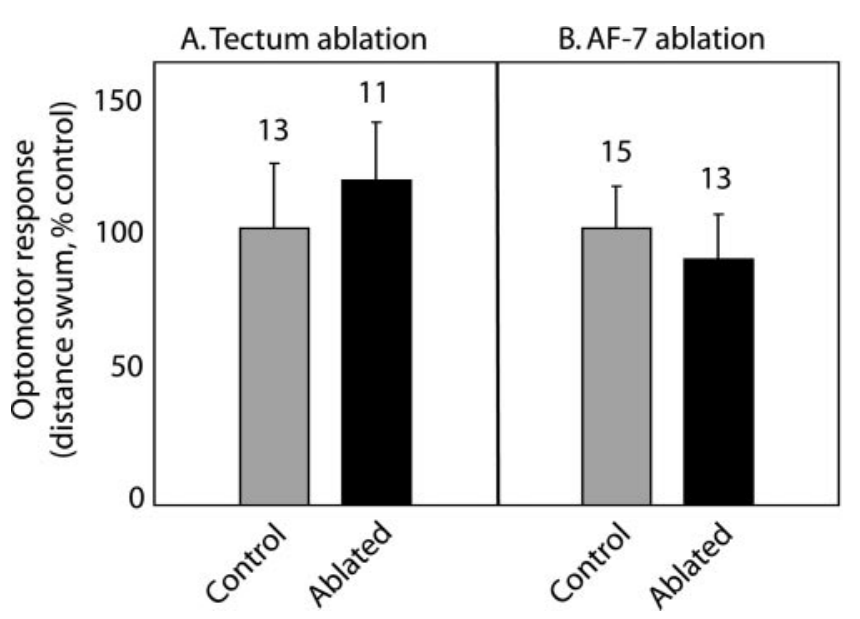

Figure 7. Effect of ablation of tectal neuropil $(A)$ and of $A F-7(B)$ on the OMR. The magnitudes of the OMR are expressed as percentage \pm SEM of control (sham-ablated). The average distances swum by control fish in $30 \mathrm{sec}$ were $4.7 \mathrm{~cm}$ for experiment $A$ and $8.2 \mathrm{~cm}$ for experiment B. Neither tectum ablation nor AF-7 ablation noticeably affected the OMR.

called visual grasp reflex (Akert, 1949; Meyer et al., 1970; Yager et al., 1977; Salas et al., 1997). A tectal role in orienting responses, toward prey and away from predators, is also supported by studies in amphibians, reptiles, birds, and mammals (Ingle, 1973; Bass, 1977; Ewert, 1984). In goldfish, the tectum appears to be required for some visual discrimination tasks (Yager et al., 1977; Davis and Klinger, 1987), whereas in sharks, it appears to be dispensable for such a behavior (Graeber et al., 1973). In contrast, an intact tectum is not required for visual background adaptation (Dijkgraaf, 1949; Gentle, 1971; confirmed in this study), phototaxis (Kicliter, 1973; Ullén et al., 1997), dorsal light response (Springer, 1977; Mori, 1993; Ullén et al., 1997), OKR (Springer et al., 1977; this study), OMR (Stehouwer, 1987; this study), and the detection of stationary barriers (Ingle, 1973) in a variety of vertebrates. On the basis of the cumulative evidence, we posit that the tectum-ablated zebrafish investigated in our study have not been challenged with the appropriate tests to reveal their deficit.

\section{Functional neuroanatomy by GFP-guided laser ablations in transgenic zebrafish}

Because of its small size and transparency, the zebrafish larva is uniquely suited to perform GFP-guided tissue ablations by laser irradiation. Laser ablations have been applied with great success in the nematode Caenorhabditis elegans (Bargmann and Avery, 1995), but application of this powerful technique in vertebrates has been hampered by the difficulty of visualizing the target. Until now, laser ablations in zebrafish behavioral studies had been restricted to large identified cells in the reticulospinal system, which were prelabeled by fluorescent dye injection (Liu and Fetcho, 1999; Gahtan and O'Malley, 2001). In this study, we used the shh::gfp transgenic line, in which the target structure was marked by a genetically encoded fluorescent reporter.

GFP-guided laser ablation may be useful for the study of other areas in the zebrafish brain, depending on the availability of celltype or area-specific enhancer sequences. However, there remain technical limitations. Deep structures $(>100 \mu \mathrm{m})$ are difficult, or impossible, to disrupt by the laser used here, probably attributable to attenuation of laser energy by absorption and scatter as the beam passes through water and biological tissue. For example, the optic chiasm could not be destroyed after $3 \mathrm{dpf}$, although transient bleaching by laser irradiation could be observed at all stages (our unpublished observations). Furthermore, areas that are close to major blood vessels are difficult targets because disruption of vasculature (which absorbs the laser light very strongly) leads to spreading tissue damage and frequently to death of the larva. Despite these limitations, laser ablations in GFP transgenic lines will increasingly complement genetic screens (Brockerhoff et al., 1995, 1998; Neuhauss et al., 1999; Baier, 2000) for which zebrafish are already a popular model.

\section{References}

Adelson EH, Bergen JR (1985) Spatiotemporal energy models for the perception of motion. J Opt Soc Am A 2:284-299.

Akert K (1949) Der visuelle Greifreflex. Helv Physiol Acta 7:112.

Baier H (2000) Zebrafish on the move: towards a behavior-genetic analysis of vertebrate vision. Curr Opin Neurobiol 10:451-455.

Baker Jr CL (1999) Central neural mechanisms for detecting second-order motion. Curr Opin Neurobiol 9:461-466.

Bargmann CI, Avery L (1995) Laser killing of cells in Caenorhabditis elegans. Methods Cell Biol 48:225-250.

Bass AH (1977) Effects of lesions of optic tectum on ability of turtles to locate food stimuli. Brain Behav Evol 14:251-260.

Brockerhoff SE, Hurley JB, Janssen-Bienhold U, Neuhauss SC, Driever W, Dowling JE (1995) A behavioral screen for isolating zebrafish mutants with visual system defects. Proc Natl Acad Sci USA 92:10545-10549.

Brockerhoff SE, Dowling JE, Hurley JB (1998) Zebrafish retinal mutants. Vision Res 38:1335-1339.

Burrill JD, Easter Jr SS (1994) Development of the retinofugal projections in the embryonic and larval zebrafish (Brachydanio rerio). J Comp Neurol 346:583-600.

Cavanagh P, Mather G (1989) Motion: the long and short of it. Spat Vis 4:103-129.

Cazin L, Precht W, Lannou J (1980) Pathways mediating optokinetic responses of vestibular neurons in the cat. Pflügers Arch 384:19-29.

Chubb C, Sperling G (1988) Drift-balanced random stimuli: a general basis for studying non-Fourier motion perception. J Opt Soc Am A 5:1986-2007.

Collewijn H (1975) Oculomotor areas in the rabbits midbrain and pretectum. J Neurobiol 6:3-22.

Davis RE, Klinger PD (1987) Spatial discrimination in goldfish following bilateral tectal ablation. Behav Brain Res 25:255-260.

Debski EA, Cline HT (2002) Activity-dependent mapping in the retinotectal projection. Curr Opin Neurobiol 12:93-99.

Demb JB, Zaghloul K, Sterling P (2001) Cellular basis for the response to second-order motion cues in Y retinal ganglion cells. Neuron 32:711-721.

Dijkgraaf S (1949) Lokalisationsversuche am Fischgehirn. Experientia 5:44-45.

Easter Jr SS (1972) Pursuit eye movements in goldfish (Carassius auratus). Vision Res 12:673-688.

Easter Jr SS, Nicola GN (1996) The development of vision in the zebrafish (Danio rerio). Dev Biol 180:646-663.

Easter SS, Schmidt JT, Leber SM (1978) Induced ipsilateral retinal projection in goldfish. J Embryol Exp Morphol 45:145-159.

Engert F, Tao HW, Zhang LI, Poo MM (2002) Moving visual stimuli rapidly induce direction sensitivity of developing tectal neurons. Nature 419:470-475.

Ewert JP (1984) Tectal mechanisms that underlie prey-catching and avoidance behaviors in toads. In: Comparative neurology of the optic tectum (Vanegas H, ed), pp 247-416. New York: Plenum.

Flandrin JM, Jeannerod M (1981) Effects of unilateral superior colliculus ablation on oculomotor and vestibulo-ocular responses in the cat. Exp Brain Res 42:73-80.

Gahtan E, O'Malley DM (2001) Rapid lesioning of large numbers of identified vertebrate neurons: applications in zebrafish. J Neurosci Methods 108:97-110.

Gentle MJ (1971) The central nervous control of colour change in the minnow (Phoxinus Phoxinus L.). I. Blinding and effects of tectal removal on normal and blind fish. J Exp Biol 54:83-91.

Gibbs MA, Northmore DP (1996) The role of torus longitudinalis in equilibrium orientation measured with the dorsal light reflex. Brain Behav Evol 48:115-120.

Gioanni H, Rey J, Villalobos J, Richard D, Dalbera A (1983) Optokinetic 
nystagmus in the pigeon (Columba livia). II. Role of the pretectal nucleus of the accessory optic system (AOS). Exp Brain Res 50:237-247.

Gnügge L, Schmid S, Neuhauss SC (2001) Analysis of the activity-deprived zebrafish mutant macho reveals an essential requirement of neuronal activity for the development of a fine-grained visuotopic map. J Neurosci 21:3542-3548.

Graeber RC, Ebbesson SO, Jane JA (1973) Visual discrimination in sharks without optic tectum. Science 180:413-415.

Harris LR, Smith AT (1992) Motion defined exclusively by second-order characteristics does not evoke optokinetic nystagmus. Vis Neurosci 9:565-570.

Hikosaka O, Wurtz RH (1983) Effects on eye movements of a GABA agonist and antagonist injected into monkey superior colliculus. Brain Res 272:368-372.

Hogben L, Slome D (1931) The pigmentary effector system. VI. The dual character of endocrine co-ordination in amphibian color change. Proc $\mathrm{R}$ Soc Lond B Biol Sci 108:10-53.

Holt CE, Harris WA (1998) Target selection: invasion, mapping and cell choice. Curr Opin Neurobiol 8:98-105.

Ingle D (1973) Two visual systems in the frog. Science 181:1053-1055.

Katte O, Hoffmann KP (1980) Direction specific neurons in the pretectum of the frog (Rana esculenta). J Comp Physiol 140:53-57.

Kay JN, Finger-Baier KC, Roeser T, Staub W, Baier H (2001) Retinal ganglion cell genesis requires lakritz, a zebrafish atonal homolog. Neuron 30:725-736.

Kicliter E (1973) Flux, wavelength and movement discrimination in frogs. Forebrain and midbrain contributions. Brain Behav Evol 8:340-365.

Kishida R (1979) Comparative study on the teleostean optic tectum. Lamination and cytoarchitecture. J Hirnforsch 20:57-67.

Klar M, Hoffmann KP (2002) Visual direction-selective neurons in the pretectum of the rainbow trout. Brain Res Bull 57:431-433.

Lazar G (1973) Role of the accessory optic system in the optokinetic nystagmus of the frog. Brain Behav Evol 5:443-460.

Lazar G, Alkonyi B, Toth P (1983) Re-investigation of the role of the accessory optic system and pretectum in the horizontal optokinetic head nystagmus of the frog-lesion experiments. Acta Biol Hungarica 34:385-393.

Ledgeway T, Smith AT (1994) The duration of the motion aftereffect following adaptation to first-order and second-order motion. Perception 23:1211-1219.

Liu KS, Fetcho JR (1999) Laser ablations reveal functional relationships of segmental hindbrain neurons in zebrafish. Neuron 23:325-335.

Lu ZL, Sperling G (1995) The functional architecture of human visual motion perception. Vision Res 35:2697-2722.

Meyer DL, Schott D, Schaefer KP (1970) Reizversuche im Tectum opticum freischwimmender Kabeljaue bzw. Dorsche (Gadus morrhua). Pflügers Arch 314:240-252.

Montgomery N, Fite KV, Taylor M, Bengston L (1982) Neural correlates of optokinetic nystagmus in the mesencephalon of Rana pipiens: a functional analysis. Brain Behav Evol 21:137-150.

Mori S (1993) Localization of extratectally evoked visual response in the corpus and valvula cerebelli in carp, and cerebellar contribution to "dorsal light reaction" behavior. Behav Brain Res 59:33-40.

Neuhauss SC, Biehlmaier O, Seeliger MW, Das T, Kohler K, Harris WA, Baier H (1999) Genetic disorders of vision revealed by a behavioral screen of 400 essential loci in zebrafish. J Neurosci 19:8603-8615.

Neumann CJ, Nüsslein-Volhard C (2000) Patterning of the zebrafish retina by a wave of sonic hedgehog activity. Science 289:2137-2139.

Orger MB, Smear MC, Anstis SM, Baier H (2000) Perception of Fourier and non-Fourier motion by larval zebrafish. Nat Neurosci 3:1128-1133.

Pasik T, Pasik P, Bender MB (1966) The superior colliculi and eye movements. An experimental study in the monkey. Arch Neurol 15:420-436.

Salas C, Herrero L, Rodriguez F, Torres B (1997) Tectal codification of eye movements in goldfish studied by electrical microstimulation. Neuroscience 78:271-288.

Scudder CA, Kaneko CS, Fuchs AF (2002) The brainstem burst generator for saccadic eye movements: a modern synthesis. Exp Brain Res 142:439-462.

Sin WC, Haas K, Ruthazer ES, Cline HT (2002) Dendrite growth increased by visual activity requires NMDA receptor and Rho GTPases. Nature 419:475-480.

Springer AD, Easter Jr SS, Agranoff BW (1977) The role of the optic tectum in various visually mediated behaviors of goldfish. Brain Res 128:393-404.

Stehouwer DJ (1987) Effect of tectotomy and decerebration on spontaneous and elicited behavior of tadpoles and juvenile frogs. Behav Neurosci 101:378-384.

Stuermer CA (1988) Retinotopic organization of the developing retinotectal projection in the zebrafish embryo. J Neurosci 8:4513-4530.

Ullén F, Deliagina TG, Orlovsky GN, Grillner S (1997) Visual pathways for postural control and negative phototaxis in lamprey. J Neurophysiol 78:960-976.

Vaina LM, Cowey A (1996) Impairment of the perception of second order motion but not first order motion in a patient with unilateral focal brain damage. Proc R Soc Lond B Biol Sci 263:1225-1232.

von Frisch K (1911) Beiträge zur Physiologie der Pigmentzellen in der Fischhaut. Pflügers Arch 138:319-387.

Wilson HR, Ferrera VP, Yo C (1992) A psychophysically motivated model for two-dimensional motion perception. Vis Neurosci 9:79-97.

Wullimann MF (1994) The teleostean torus longitudinalis: a short review on its structure, histochemistry, connectivity, possible function and phylogeny. Eur J Morphol 32:235-242.

Wurtz RH, Goldberg ME (1972) Activity of superior colliculus in behaving monkey. IV. Effects of lesions on eye movements. J Neurophysiol 35:587-596.

Yager D, Sharma SC, Grover BG (1977) Visual function in goldfish with unilateral and bilateral tectal ablation. Brain Res 137:267-275.

Zhang LI, Tao HW, Holt CE, Harris WA, Poo M (1998) A critical window for cooperation and competition among developing retinotectal synapses. Nature 395:37-44. 